\title{
Understanding Chemical Reactions beyond Transition-State Theory
}

\author{
Jeremy O. Richardson*
}

\begin{abstract}
Transition-state theory is one of the most successful theories in chemistry. Not only does it provide a simple formula for computing the rate of a reaction but it defines our understanding of how a chemical reaction occurs - by overcoming an energy barrier. However, the theory is based on classical assumptions and ignores several quantum effects which can be important for certain reactions, particularly those involving hydrogen transfer at low temperatures. We are developing a more general quantum version of transition-state theory which reveals how quantum tunnelling and nonadiabatic effects can strongly affect the rate.
\end{abstract}

Keywords: Instanton · Nonadiabatic · Path integral · Quantum · Rate · Ring polymer · Transition-state theory · Tunnelling

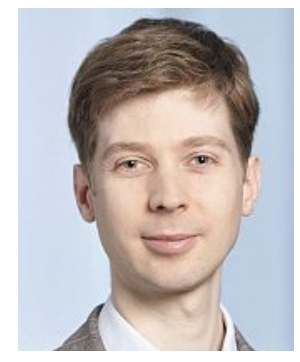

\section{Introduction}

The role of theoretical chemistry is to provide understanding of fundamental chemical processes. With a thorough understanding of molecular systems, scientists are able to explain experimental observations, make accurate predictions of the behaviour of new systems, and hence design them to have certain properties of interest.

In the majority of cases, it is not neces-

Jeremy Richardson was born in Cardiff and studied at Cambridge University where he also completed his $\mathrm{PhD}$ under the supervision of Stuart Althorpe. He was a postdoc and Humboldt Research Fellow at Friedrich-Alexander University ErlangenNürnberg in the group of Michael Thoss and a Junior Research Fellow at Durham University. In September 2016 he moved to ETH Zurich as the Assistant Professor of Theoretical Molecular Quantum Dynamics in the Laboratory of Physical Chemistry.

${ }^{*}$ Correspondence: Prof. Dr. J. O. Richardson ETH Zurich, $\mathrm{HCl}$ D267.3

Vladimir-Prelog-Weg 2, CH-8093 Zurich

E-mail: jeremy.richardson@phys.chem.ethz.ch acquires more energy and is more likely to overcome the barrier; thus the rate increases. Alternatively the rate can also increase if a catalyst is employed to reduce the barrier. This equation is therefore at the heart of our understanding of a wide range of chemical reactions. It explains why we heat reactions to make them faster and how enzymes can control biochemistry.

Nonetheless, Arrhenius' equation was obtained by hypothesis backed up by experimental observations but was not derived from first principles. It could not therefore explain how the activation energy depends on the specific molecules under consideration or determine when certain chemical reactions might deviate from this behaviour. Since the advent of quantum mechanics, the underlying theory describing the behaviour of molecules is completely known. ${ }^{[2]}$ Schrödinger's equation gives one representation for the laws of quantum mechanics by describing the dynamics of a molecule as a time-dependent wave in a high-dimensional space. These equations are unfortunately practically impossible to solve for all but the smallest molecules and even then present results in a form which is difficult to interpret. One does not want to artificially reduce the number of degrees of freedom as this approach cannot account for the complexity of chemistry, whereby the molecular properties can be dramatically changed with only a small variation in the functional groups. It is thus necessary to make certain approximations in order to explain molecular behaviour. When new theories are based solely on rigorous approximations to quantum mechanics, as opposed to using phenomenological observations, it is termed an ab initio method.

Transition-state theory (TST) is a remarkably successful $a b$ initio method for determining the rate of a chemical reaction. 
Eyring, [3a] Evans and Polyani[3b] derived a formula equivalent to Eqn. (1). They show that the activation energy is given by the energy difference between a transition state and the reactants and that the prefactor, $A$, is dominated by quantum zero-point energy contributions. Not only does TST provide us with a numerical method for obtaining the rate of a chemical reaction based on a limited knowledge of the potential-energy surface, but even more importantly, it provides us with an understanding of how chemical reactions take place. The activation energy has an enthalpic contribution due to the breaking of bonds as well as an entropic contribution associated with conformational changes necessary for the atoms to rearrange.

As noted by Wigner, ${ }^{[4]}$ TST is based on classical assumptions rather than the true dynamics of atoms and molecules and several quantum effects are ignored. These include tunnelling and nonadiabatic effects, which for certain reactions can dominate the process. It is not possible to reintroduce the quantum effects back into classical TST in a simple way because all degrees of freedom are coupled together. ${ }^{[5]}$ Therefore if we are to understand how these chemical reactions proceed, then a more general formulation of rate theory is needed.

A major theme of our research is the derivation of new theories to predict the rate of a chemical reaction without ignoring these quantum effects. We search for a good compromise, to obtain as much accuracy as possible, while retaining the simplicity of the TST approach. As well as giving a computationally feasible numerical method, this has the advantage of giving a simpler theory which is much easier to interpret.

\section{Quantum Tunnelling}

One of the most important effects neglected by TST is quantum tunnelling. It is well known that tunnelling plays a role in many reactions, ${ }^{[6]}$ especially where hydrogen is involved, which being so light strongly manifests its quantum nature. Tunnelling is an essentially non-classical process in which a particle can cross a barrier even if its energy is lower that that of the barrier height. This process is represented in Fig. 1.

Although no classical trajectory exists which tunnels, the quantum dynamics of the particle can be approximated using socalled 'semiclassical' trajectories. Using the equation, $E=p^{2} / 2 m+V(x)$, one can see that if the particle energy, $E$, is lower than the potential energy, $V(x)$, then the kinetic energy term, $p^{2} / 2 m$, must be negative. Because the mass, $m$, is positive, this implies that the momentum, $p$, is imagi-



Fig. 1. Representation of a tunnelling process described by a semiclassical trajectory (blue/red line). Configurations, $x$, on the left of the potential-energy barrier, $V(x)$, are defined as 'reactants' and configurations on the right are 'products'. Because the total energy, $E$, is less than the barrier height, $V^{\ddagger}$, the trajectory is required to pass through a classically-forbidden region marked in red. In this region the trajectory travels in imaginary time.

nary and that therefore particles tunnelling through barriers can be considered to be moving in imaginary time. ${ }^{[7]}$

Starting from the exact definition for the rate of a chemical reaction, ${ }^{[8]}$ and by carefully following through a mathematical derivation based on taking semiclassical approximations, ${ }^{[9]}$ we arrive at the following approximation:

$$
k=A \mathrm{e}^{-S / \hbar}
$$

This formula relates the rate to the 'action', $S$, along the optimal tunnelling pathway known as an 'instanton'. [7,10,11] The instanton is an orbit with periodicity of imaginary time $\beta \hbar$ and is represented by the red line in Fig. 1. The action can be defined as $S=W+\hbar \beta E$ where $W=\oint \sqrt{2 m[V(x)-E]} \mathrm{d} x$ is an integral around the orbit. The prefactor, $A$, is determined by fluctuations of the action around the instanton.

At high temperatures, the period is short and the tunnelling pathway skims the top of the barrier giving $S=\beta \hbar V^{\ddagger}$. In this case the classical TST formula, Eqn. (1), is recovered, which shows that tunnelling is not important at high temperatures. However, at low temperatures, the dominant pathway exists as an orbit at energy less than the barrier height, i.e. $E<V^{\ddagger}$, which implies a tunnelling process. Then $S<\beta \hbar V^{\ddagger}$, and the rate deviates significantly from the TST prediction and can often be orders of magnitude faster. In this way the instanton approach predicts rate constants along with their mechanisms. In certain cases there is a competition between different mechanisms and the instanton approach is able to justify why one is preferred - the one with the smallest $S$. This may not always be the mechanism with the lowest barrier, as due to tunnelling effects the rate through a tall, narrow barrier can be faster than that over a short, wide barrier.[12]

In order to apply the method to predict rates and mechanisms of chemical reactions, it is necessary to obtain the tunnelling pathway in multidimensional space. Atoms exist in a three-dimensional space and therefore a molecule of $N$ atoms has $3 N$ degrees of freedom. We have developed the ring-polymer instanton method ${ }^{[13]}$ for locating the optimal tunnelling pathway in many dimensions using a discretization of the path and by locating stationary points of $S$. The potential energy along the pathway is evaluated explicitly and not fit to any predetermined shape or based on a Taylor expansion about the transition state. Although in the instanton method the modes perpendicular to the pathway are treated within a harmonic approximation, they are not ignored and do at least contribute with a zero-point energy effect. This makes the instanton method the stateof-the-art approach for computing reaction rates in polyatomic systems exhibiting tunnelling effects.

As a simple example to a polyatomic chemical reaction, we applied the method to compute the rate of the benchmark gasphase reaction $\mathrm{H}+\mathrm{CH}_{4} \rightarrow \mathrm{H}_{2}+\mathrm{CH}_{3}$. The mechanism is shown in Fig. 2. We were able to take advantage of high-accuracy on-the-fly potential-energy surface calculations ${ }^{[14]}$ and our results suggest that the major source of error typically comes from the electronic-structure calculation of the potential rather than from the instanton approximation. Therefore it will usually be 


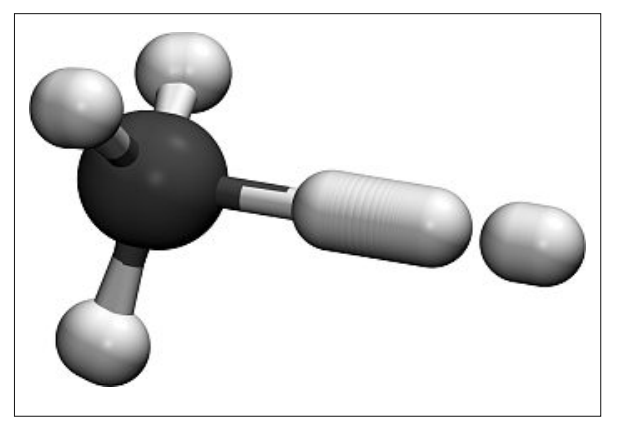

Fig. 2. Representation of the ring-polymer instanton describing proton tunnelling in the $\mathrm{H}+\mathrm{CH}_{4}$ reaction at $200 \mathrm{~K}$. All atoms take part to some extent in the tunnelling process and become delocalized as they pass through the potential barrier. Reproduced from ref. [14].

better to use the efficient instanton method on an accurate surface rather than more computationally time-consuming quantum dynamics on a less-accurate but cheaper potential-energy surface.

It is also possible to extend the approach to compute a microcanonical reaction rate. ${ }^{[15]} \mathrm{A}$ simple and accurate microcanonical instanton theory will be of particular importance for simulating the complex reaction networks which occur in atmospheric chemistry. In these systems, thermal equilibrium is often not achieved due to low pressure conditions and the existence of reactions with pre-reactive complexes, ${ }^{[16]}$ and thus energy-dependent unimolecular rates are needed in simulations.

It is well known that tunnelling can be important for the formation and dissociation of molecules in the atmosphere and there already are experiments showing strong tunnelling effects in a number of atmospheric and astrochemical reactions. ${ }^{[17,18]}$ A reliable theoretical method is required to elucidate the mechanism for these processes and make predictions for the rate of each step in a complex reaction network, which is hard to study experimentally. The microcanonical instanton method provides an efficient and accurate approach for computing these rate constants from first principles and we intend to apply it to simulate the experimental findings and test the conclusions. The need for this theory is even more extreme in astrochemistry where the molecules tend to be found at even lower temperatures and pressures. The same methodology can be applied to reactions of relevance to this field. Accurate rate constants are needed for modelling the chemistry in the earth's atmosphere as well as in space. By including tunnelling effects correctly into these models, we hope to obtain a better understanding of the earth's climate and of interstellar chemistry.

We have also used instanton theory to study the hydrogen-bond dynamics of molecular clusters including water clusters ${ }^{[19]}$

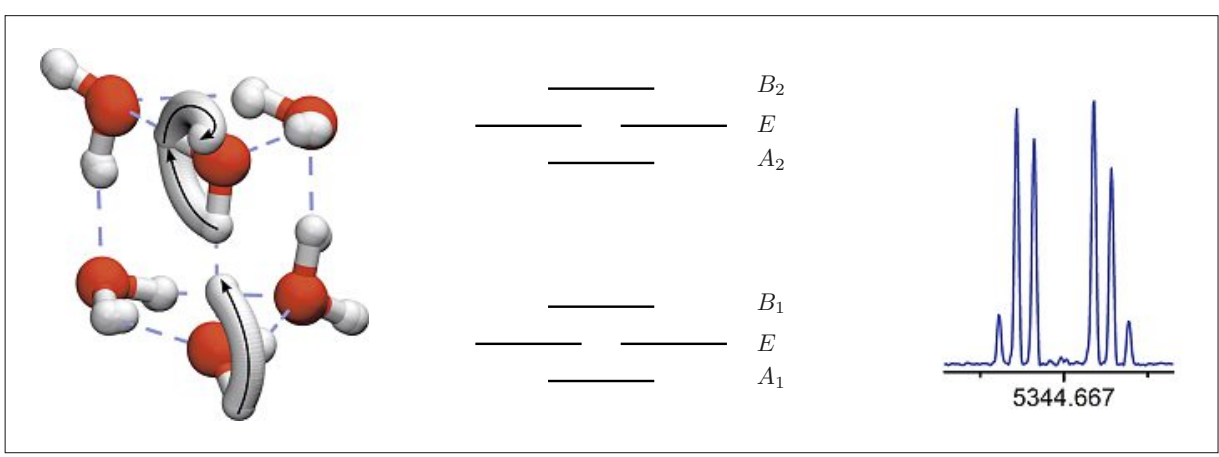

Fig. 3. The optimal tunnelling pathway calculated for the water hexamer prism (left) showing concerted hydrogen-bond breaking. The instanton method predicts the correct splitting pattern (a doublet of triplets, middle) as observed in experiment (right).

and the formic acid dimer.[20] Our study of the water hexamer prism ${ }^{[21 b]}$ shows clearly that it would not have been possible to know the tunnelling pathways a priori as they lie far from the minimum-energy paths and as shown in Fig. 3 unexpectedly allow two hydrogen bonds to break simultaneously. The predictions were in line with the experimental spectroscopy measurements ${ }^{[21]}$ and explain the observed doublet-of-triplet splitting patterns. The greatest advantage of the instanton approach to studying tunnelling effects is its scalability. Applying the method itself has a negligible computational cost even for large systems and the only expensive part is the evaluation of the potential-energy surface. This of course dominates the computational expense for most molecular simulation techniques including classical or path-integral molecular dynamics. Due to advancements in computational power, these are now commonly applied with an on-the-fly evaluation of the forces, typically using density functional theory. ${ }^{[22,23]}$ However, the optimization of the instanton will typically require far fewer evaluations of the potential-energy surface than other simulation techniques which require a statistically large number of samples. We thus expect the instanton methodology to also make an impact on the study of tunnelling processes in the condensed phase. ${ }^{[24-26]}$ In particular we intend to continue to study the rearrangement dynamics of hydrogenbond networks in large systems including water clusters adsorbed on surfaces and ice crystals.

\section{Nonadiabatic Effects}

Our understanding of many chemical reactions is based on the Born-Oppenheimer approximation, which implies that the dynamics proceeds on a single adiabatic potential-energy surface. ${ }^{[4]}$ Deviations from this behaviour are known as nonadiabatic effects. Typical examples of nonadiabatic reactions include electron transfers, ${ }^{27]}$ proton-coupled electron transfers ${ }^{[28]}$ and photoexcited internal conversion.

Chemical reactions involving electron transfer occur in many different environments, from redox reactions to photosynthesis and the harvesting of light in solar cells. ${ }^{[29]}$ In these reactions, an electron is transferred from a donor to an acceptor; these entities can be anything from small solvated ions ${ }^{[30]}$ to large protein complexes. ${ }^{[31]}$ Thus there are at least two important electronic states involved in the reaction and typically the Born-Oppenheimer approximation breaks down, making it necessary to consider nonadiabatic dynamics in order to describe and predict the rate of the process.

The simplest electron-transfer reactions are commonly described using Marcus theory.[29] Like TST, Marcus theory neglects tunnelling effects and most quantum-mechanical extensions are only valid for a spin-boson model of the process. ${ }^{[32]}$ We have developed an instanton theory for describing electron-transfer reactions including these quantum effects in a more general multidimensional anharmonic environment. ${ }^{[33]}$ Its form is given again by Eqn. (2). Here the nuclei travel along an optimal tunnelling pathway which is partly on the reactant potentialenergy surface and partly on the product surface. The electron-transfer process occurs simultaneously with the nuclear tunnelling event. The rate thus depends not only on the height and width of the barrier but also on the coupling between the reactant and product electronic states. In the high-temperature limit, the instanton reduces to the classical rate and to Marcus theory when the system has the form of the spin-boson model.

Only simple numerical algorithms including a saddle-point optimization are required to apply the method in practice and it is therefore computationally inexpensive. Recent work applied both classical and semiclassical methods to an asymmet- 
ric system-bath model with anharmonic free-energy curves in order to explore the behaviour of the rates of various approaches with respect to both anharmonicity and tunnelling. ${ }^{[34]}$ It was found that in certain cases the rate predicted by the standard Marcus theory was in error by orders of magnitude but that the instanton approach was in error by no more than $1 \%$.

Anharmonic effects cannot be described by methods based on the spin-boson model which effectively uses a global harmonic approximation to map the system onto a set of harmonic oscillators. In contrast, the instanton approach locates the optimal tunnelling pathway on the full potential-energy surface but then uses a local harmonic approximation round this configuration to obtain the fluctuation factor. This can be a valid approximation for gas-phase or solid systems but is not directly applicable to liquid systems, which comprise some of the most interesting and relevant examples of electron transfer. The reason for this is that many different but similar transition states exist corresponding to minor rearrangements of the outer solvation sphere and therefore even a local harmonic approximation breaks down. A more general method would be required to study such systems which samples the different instantons and makes no harmonic approximations. A currently running project in our group is concerned with generalizing this instanton approach to obtain a path-integral sampling method which can be used to study solvated electron transfer.

In other projects we are developing new approaches for simulation of nonadiabatic dynamics based on classical trajectories in an extended space allowing them to move between potential-energy surfaces. ${ }^{[35]}$ This will be used to describe photoexcited reactions and spectroscopy. ${ }^{[36]}$ Using a combi- nation of these approaches, we will study tunnelling and nonadiabatic effects occurring in a wide range of chemical reactions; something which would not have been possible with transition-state theory alone.

\section{Received: February 19, 2018}

[1] S. Arrhenius, Z. Phys. Chem. 1889, 4, 226.

[2] P. A. M. Dirac, Proc. R. Soc. Lond. A 1929, 123, 714

[3] a) H. Eyring, J. Chem. Phys. 1935, 3, 107; b) M. G. Evans, M. Polanyi, Trans. Faraday Soc. 1935, 31, 875 .

[4] E. Wigner, Trans. Faraday Soc. 1938, 34, 29

[5] W. H. Miller, Acc. Chem. Res. 1993, 26, 174

[6] R. P. Bell, 'The Tunnel Effect in Chemistry', Chapman and Hall, London, 1980

[7] W. H. Miller, J. Chem. Phys. 1975, 62, 1899.

[8] W. H. Miller, S. D. Schwartz, J. W. Tromp, J. Chem. Phys. 1983, 79, 4889

[9] J. O. Richardson, J. Chem. Phys. 2016, 144, 114106.

[10] S. Coleman, 'The uses of instantons', in: Proc. Int. School of Subnuclear Physics; also in S. Coleman, 'Aspects of Symmetry', chap. 7, pp. 265-350, Cambridge University Press, 1985 Erice, 1977

[11] V. A. Benderskii, D. E. Makarov, C. A. Wight. 'Chemical Dynamics at Low Temperatures', vol. 88. Adv. Chem. Phys. Wiley, New York, 1994.

[12] B. K. Carpenter, Science 2011, 332, 1269.

[13] J. O. Richardson, S. C. Althorpe, J. Chem. Phys. 2009, 131, 214106.

[14] A. N. Beyer, J. O. Richardson, P. J. Knowles, J. Rommel, S. C. Althorpe, J. Phys. Chem. Lett. 2016, 7, 4374 .

[15] J. O. Richardson, Faraday Discuss. 2016, 195, 49.

[16] S. Álvarez-Barcia, M.-S. Russ, J. Meisner, J. Kästner, Faraday Discuss. 2016, 195, 69.

[17] R. J. Shannon, M. A. Blitz, A. Goddard, D. E. Heard, Nat. Chem. 2013, 5, 745.

[18] N. M. Kidwell, H. Li, X. Wang, J. M. Bowman, M. I. Lester, Nat. Chem. 2016, 8, 509.

[19] a) J. O. Richardson, S. C. Althorpe, D. J Wales, J. Chem. Phys. 2011, 135, 124109; b) J. O. Richardson, D. J. Wales, S. C. Althorpe, R. P. McLaughlin, M. R. Viant, O. Shih, R. J. Saykally, J. Phys. Chem. A 2013, 117, 6960.
[20] J. O. Richardson, Phys. Chem. Chem. Phys. 2017, 19, 966.

[21] a) C. Pérez, M. T. Muckle, D. P. Zaleski, N. A. Seifert, B. Temelso, G. C. Shields, Z. Kisiel, B. H. Pate, Science 2012, 336, 897; b) J. O. Richardson, C. Pérez, S. Lobsiger, A. A. Reid, B. Temelso, G. C. Shields, Z. Kisiel, D. J. Wales, B. H. Pate, S. C. Althorpe, Science 2016, $351,1310$.

[22] D. Marx, M. Parrinello, J. Chem. Phys. 1996, 104, 4077.

[23] a) M. Ceriotti, J. Cuny, M. Parrinello, D. E. Manolopoulos, Proc. Natl. Acad. Sci. USA 2013, 110, 15591; b) M. Rossi, M. Ceriotti, D. E. Manolopoulos, J. Phys. Chem. Lett. 2016, 7, 3001.

[24] H. Jónsson, Proc. Natl. Acad. Sci. USA 2011, 108, 944.

[25] a) J. B. Rommel, Y. Liu, H.-J. Werner, J. Kästner, J. Phys. Chem. B 2012, 116, 13682; b) T. Lamberts, J. Kästner, Astrophys. J. 2017, 846, 43.

[26] W. Fang, J. O. Richardson, J. Chen, X.-Z. Li, A. Michaelides, Phys. Rev. Lett. 2017, 119, 126001.

[27] D. Chandler. 'Electron transfer in water and other polar environments, how it happens', in: 'Classical and Quantum Dynamics in Condensed Phase Simulations', Eds. B. J. Berne, G. Ciccotti, D. F. Coker, World Scientific, Singapore, 1998. Chap. 2, p. 25.

[28] S. Hammes-Schiffer, J. Am. Chem. Soc. 2015 , 137,8860 .

[29] R. A. Marcus, Rev. Mod. Phys. 1993, 65, 599.

[30] R. A. Kuharski, J. S. Bader, D. Chandler, M. Sprik, M. L. Klein, R. W. Impey, J. Chem. Phys. 1988, $89,3248$.

[31] J. Blumberger, Phys. Chem. Chem. Phys. 2008, $10,5651$.

[32] P. Siders, R. Marcus, J. Am. Chem. Soc. 1981, 103, 741 .

[33] a) J. O. Richardson, R. Bauer, M. Thoss, J. Chem. Phys. 2015, 143, 134115; b) J. O. Richardson, J. Chem. Phys. 2015, 143, 134116.

[34] J. Mattiat, J. O. Richardson, J. Chem. Phys. 2018, 148, 102311

[35] a) H.-D. Meyer, W. H. Miller, J. Chem. Phys. 1979, 70, 3214; b) G. Stock, M. Thoss, Phys. Rev. Lett. 1997, 78, 578; c) J. O. Richardson, M. Thoss, J. Chem. Phys. 2013, 139, 031102.

[36] J. O. Richardson, P. Meyer, M.-O. Pleinert, M. Thoss, Chem. Phys. 2017, 482, 124. 